\title{
Le bon air des Alpes. Entre histoire culturelle et géographie des
} représentations. Présentation

\section{Claude Reichler}

\section{Citer ce document / Cite this document :}

Reichler Claude. Le bon air des Alpes. Entre histoire culturelle et géographie des représentations. Présentation. In: Revue de géographie alpine, tome 93, $n^{\circ} 1,2005$. Le bon air des Alpes. pp. 9-14;

doi : 10.3406/rga.2005.2327

http://www.persee.fr/doc/rga_0035-1121_2005_num_93_1_2327

Document généré le 06/06/2016 


\title{
Le bon air des Alpes \\ Entre histoire culturelle et géographie des représentations Présentation
}

\author{
Claude Reichler
}

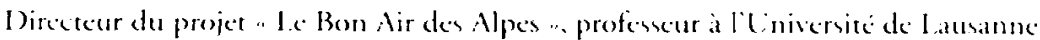

C.latude. Reichler(ar unil.ch

\section{La bibliographie est présentée en page 20}

$\mathrm{L}$ e dossier que nous présentons dans ce numéro est le fruit d'une recherche interdiscipliSuisse. Suisse'. L'objectif de notre projer était double : 1 ) évaluer la place et l'importance que prend l'air, l'image de l'air et sa réputation bénéfique, pour les habitants et les usagers des Alpes aujourd'hui ; 2) retracer l'histoire de cette question, c'est-à-dire l'histoire des perceptions, des représentations et des usages de l'air des Alpes, depuis le début du XIX siècle environ.

Le second point a pris pour nous, pour l'instant du moins, plus d'importance que le premier dans une recherche dont le centre est constitué par des sciences humaines et sociales. Plus que d'autres peut-être, un thème comme celui de l'air exige la prise en considération de sa dimension historique, dès lors qu'on le traite dans une aire géographique délimitée. Comprendre ce qu'il en est de l'air des Alpes pour ses usagers aujourd'hui, c'est d'abord connaitre ce qu'il en a été dans le passé, faire l'histoire des représentations héritées, des perceptions apprises, de l'évolution des usages. L'air en effet n'est pas seulement une donnée objective, mesurable en termes de quantité ou de qualité. Il possède aussi une résonance subjective, influencée par des imaginaires parfois anciens, par des appréciations liées à l'espace habité, au contexte social, aux artentes et au symbolique. Autant que de l'air qu'on respire aujourd'hui, l'air des Alpes est composé de la mémoire de ses usages passés. Forts de la conscience d'une profondeur historique et des valeurs immatérielles qu'elle recèle et transmet, nous pouvons interroger le présent et esquisser des perspectives pour l'avenir.

Ies moyens que nous nous sommes donnés dans cette recherche sont donc, d'abord, ceux de l'enquête historique. Pour être complète, celle-ci doit se déployer dans plusicurs domaines : en histoire des sciences, de la météorologie, de la médecine, de l'architecture et des aménagements dans les stations d'altitude ; en histoire des représentations aussi, particulièrement de la peinture et de la littérature, sans oublier les récits de voyage et les guides touristiques. En même temps, notre équipe développe une réflexion en géographie et dans les "sciences du tourisme" : par l'inventaire, jamais exhaustif, des produits touristiques proposés par les stations ou les régions, ainsi que des arguments de promotion et des images

1 - PNR 48 " Paysages er habitats de l'are alpin ". ()n peut obtenir des informations sur ce programme par le site internet hup://www.pnr $48 . \mathrm{ch} /$. 
publicitaires rattachés à l'air des Alpes; par l'analyse de ces documents et de leur contexte de production (reconversion des stations, nouvelle mobilité, changement climatique...) ; par une enquête qualitative, enfin, sur les attentes et les valorisations de l'air d'altitude chez les usagers contemporains, habitants ou touristes.

Puisque la question de l'air des Alpes rejoint celle des représentations qu'on s'en est fait, et qu'on s'en fait encore, cherchons à regrouper ces représentations de manière synthétique. Je le ferai en mettant en évidence deux aspects complémentaires : l'air apparaît à la fois comme un élément du paysage et comme une fonction de la vie (humaine).

Le terme de paysage n'est pas employé ici au hasard. Pour nous, le paysage n'est pas l'affaire de la seule vision, du seul regard. I e paysage concerne tous les sens : l'ouie, l'odorat, le toucher (au sens extensif comprenant tout ce qui affecte l'épiderme), le goût même, sans oublier le sens interne, qui assure la coordination des perceptions sensorielles, ni non plus le sens cinétique, sens du mouvement dont l'importance apparaît considérable depuis les travaux d'Alain Berthoz (Berthoz, 1997)². Si l'air, conçu par toute la tradition aristotélicienne comme un des quatre éléments fondamentaux de la matière, est toujours perçu comme un tout dans l'imaginaire, il le doit pour une part à la place extrêmement importante qu'il occupe dans les opérations sensibles et mentales qui transforment l'espace en paysage à travers la perception. Il n'est donc pas suffisant de considérer l'air "en lui-même"; il faut tenir compte de son action météorologique tout entière. Vents, nuages, tempêtes, états de la lumière, de l'humidité, de la chaleur : l'air comme élément du paysage prend toutes ces formes à travers la transformation des météores. L'histoire des usages de l'air recoupe donc l'histoire de la perception des météores et, au-delà d'elle, l'histoire des représentations paysagères. On sait combien celle-ci est riche pour les Alpes, où la variable " altitude " ajoute une dimension supplémentaire, celle de l'orientation verticale et des dynamismes imaginaires puissants qui s'y rattachent.

Envisagée comme fonction de la vie humaine, la question de l'air est d'abord celle des propriétés chimiques et physiologiques de ce mélange de gaz qu'on appelle air, et qui est absolument nécessaire à la vie. Mais elle dépasse aussi ce seul cadre matériel : par les modifications qui affectent l'air, la qualité de la vie change, la santé peut être modifiéc, et avec elle toutes les relations de l'homme avec l'extérieur; le sentiment de soi, le psychisme lui-même sont touchés. Ia médecine et l'histoire de la médecine trouvent leur place ici, manifestées par le courant de pensée associé au nom d'Hippocrate, qui fonde l'action thérapeutique sur une modification des relations de l'homme avec son environnement. Plus largement encore, les diverses proprićtés de l'air (chimiques, physiques et optiques) exercent une action sur la vie humaine et peuvent en modifier les conditions. On comprend l'importance qu'ont eue pour la médecine les expériences liées aux variations des paramètres de l'air et l'effort théorique, parfois désordonné, qui a accompagné l'observation. Audelà du strict domaine thérapeutique, les propriétés de l'air sont apparues susceptibles de modifier la perception de soi en produisant les sentiments de bien-être ou de malaise qui appartiennent au vaste domaine de la météo-sensibilité (de la Soudière, 1999).

2 J'ai présenté cette théorie polysensorielle du paysage dans Reichler, 2002. V. les travaux d'Alain ('orbin sur l'histoire des sens, et aussi Corbin, 2001. 
L'air-paysage et l'air-santé constituent donc les deux versants de notre projet. Nos recherches montrent leur constante association, selon des dosages variables, dans l'histoire des perceptions, des représentations et des usages. Flles nous ont conduits aussi à proposer une périodisation dans l'histoire culturelle de l'air des Alpes, qui fait apparaître deux périodes charnières où air-paysage et air-santé, composante météorologique et composante médicale, sont noués de manière particulière dans les pratiques et les discours.

La première période est celle des découvertes scientifiques qui marquent la fin du XVIII" siècle et le début du XIX" (1780-1830 environ). La physique, la chimie, la météorologie transforment complètement la compréhension des phénomènes liés à l'air. Les recherches de Priestley, Lavoisier, Gay-Lussac, Saussure, Luke Howard, Humboldt, d'autres encore, permettent de décrire scientifiquement la respiration et la combustion, la densité et la pression barométrique, de proposer une typologie des formations nuageuses, de comprendre les effets climatiques de la latitude et de l'altitude... Cette effervescence scientifique est accompagnée d'une véritable fascination pour l'air et les métćores dans la peinture et la littérature, de même que dans l'imaginaire social en général. C'est l'époque des " cloud studies " de Constable, des "orages désirés" de Chateaubriand, des brumes de Kaspar David Friedrich, des notations météorologiques quotidiennes dans les journaux intimes... De cette période va naître un développement de l'intérêt médical pour l'air dans la duréc longue du XIX siècle. Des recherches importantes jalonnent l'histoire de la médecine; clles se concentrent sur deux objets : la phtisie d'une part, dont les ravages vont grandissants partout dans le monde, et contre laquelle les thérapies basées sur l'air et l'altitude apparaitront favorables; les modifications physiologiques dues à l'altitude d'autre part, qui vont mettre en évidence d'autres propriétés thérapeutiques dans l'environnement des régions élevées, mais aussi des symptômes d'affaiblissement, d'atonie, de nausées..., rassemblés sous le nom de " mal des montagnes ". Celui-ci suscite de nombreuses recherches expérimentales, dont les retombées parfois positives donnent lieu à des dispositifs de soins curatifs et préservatifs. Cette période est celle du mouvement continu de ce qu'on peut appeler une médicalisation de l'air d'altitude, préparée, expérimentée, effective enfin. La deuxième période nodale apparaît alors avec la fin du XIX" siècle et le début du XX" (1880-1920 environ), caractérisée par le triomphe de la cure d'altitude, de l'aérothérapie, la construction des grands sanatoriums et le développement des stations, l'apparition des pratiques de santé en plein air et des sports alpins. Ce moment est celui où apparaissent de grandes œuvres paysagères dans la peinture alpestre, qui en renouvellent complètement l'approche ${ }^{3}$; il est aussi celui où la photographie, l'affiche, le cinéma, commencent à prendre en charge les discours d'escorte portant sur les vertus de la montagne et la représentation de l'air et des météores. Il sera suivi des quelques décennies durant lesquelles la diversification des thérapies antituberculeuses se poursuit, avec un empirisme qui paraît rétrospectivement inquiétant ; finalement le succès des antibiotiques dans le traitement de la maladic jette le discrédit sur

3- Je pense aux aeuvres de Ferdinand Hodler ou de Ernst I udwig Kirchner, mais il y aurait bien d'autres exemples, notamment dans l'expressionnisme. (On notera que le roman de Thomas Mann, l.a Montagne magique parait en 1924. 
la cure sanatoriale. La seconde moitié du XX" siècle apparaît d'abord dans notre chronologie comme une époque floue, dont les tendances sont dispersées. Y prédomine la difficile reconversion des sanatoriums et des stations sanatoriales, processus durant lequel les stations dévolues à la curc ont été connotées d'une moins-value " morbidité ". Mais y apparaissent aussi de nouvelles activités touristiques, la multiplication des résidences secondaires, une valorisation grandissante, quoique ambiguë, apportée à la montagne par l'industrialisation des pratiques sportives hivernales. La période récente a vu renaître fortement l’intérêt pour l'air et les météores à travers les problèmes posés par l'écologie et la gestion de la pollution, par l'explosion de la mobilité de loisir, par les nouvelles formes du tourisme et la demande de santé émanant de catégories sociales diversifićes (les retraités, bien plus que les enfants...). Peut-être sommes-nous au cœur d'un troisième næud où se recroisent, selon des figures encore mal discernables, les différents fils qui composent l'histoire de l'air ?

Des périodes nodales et de longs entre-deux ; des moments où les interactions entre les domaines sont fortes, les emprunts et les recoupements concentrés et productifs, et des moments de développement non parallèle des champs. Ainsi, pour donner deux exemples rapides, la médecine du XIX siècle, largement soumise à la méthode expérimentale, suir les voies d'une science positive, alors qu'auparavant et de nouveau par la suite, elle dialogue avec les savoirs humanistes et avec les arts; ou le tourisme après 1950 , qui semble se propager essentiellement sur des bases économiques et de divertissement, sans grand lien avec l'esthétique du paysage. Aurions-nous là un modèle de configurations temporelles qui décrive de manière acceptable l'histoire culturelle de l'air alpin ? Nous le formulons pour l'instant comme une hypothèse de travail, qu'il faudra tester et affiner.

Je voudrais faire observer encore que durant les périodes nodales, ou bien les précédant ou les suivant de peu, une production imaginaire abondante et souvent remarquable accompagne l'intérêt scientifique, social ou économique et lui apporte un commentaire sur le plan symbolique. Est posé là le problème de l'oeuvre d'art (littérature comprise) dans ses rapports avec le contexte de son apparition. Notre projet y accorde de l'importance, donnant place à des analyses de peinture et d'architecture, des commentaires portant sur des œuvres littéraires. L'œuuve d'art constitue pour nous une trace précieuse des représentations que se sont données les hommes et les communautés du passé ; une trace au second degré, qui permet d'accéder non directement aux choses de la nature et aux objets construits par les hommes, mais aux modèles représentatifs qui transmettent les significations qu'a prises le monde pour eux, c'est-à-dire aux modes de compréhension et aux schèmes de pensée des cultures passées. Complémentairement à la science, qui énonce des lois générales et des domaines de validité, l'œuvre d'art donne à connaître la singularité d'une forme du monde ; elle en fait une singularité partagée, universalisable par l'intuition sensible et l'interprétation rationnelle. I a place que notre projet accorde à l'analyse des productions artistiques ou fictionnelles témoigne d'une conviction fondée : celle que la question de l'air ne peut pas être traitée valablement sans le concours des disciplines et la mise en relations des domaines de compétences. Aborder l'air par son histoire, par ses représentations et par ses usages demande une attitude de coopération des disciplines, une capacité à décrire et à analyser les rationalités multiples dont il relève et les pratiques diverses qui en dépendent. L'air tient de trop près à l'humain pour qu'on puisse le constituer purement en objet de 
savoir, de service médical ou de marketing touristique. Il demeure toujours pour qui l'aborde une question globale, et il est devenu aujourd'hui pour nos sociétés un problème.

I.e dossier que nous présentons dans ce numéro de la Revue de géographie alpine ne reflète évidemment qu'une partie de notre programme de recherche. S'il ne peut être complet, ce dossier ambitionne d'être représentatif, autant par les disciplines scientifiques des contributions que par les domaines de la géographie et de l'histoire culturelle que le problème de l'air concerne. Il met l'accent sur les usages de l'air dans son aspect "santé " plus que sur le paysage. I.'histoire des thérapies basées sur l'air d'altitude a été d'abord une histoire de la géographic dans ses applications médicales. À travers l'influence des recherches de Alexandre von Humboldt, Daniela Vaj décrit les ramifications de l'idée de répartition géographique des maladies, qui parcourt tout le XIX" siècle, et les spéculations et théories que cette idée a engendrées à propos du mal le plus répandu et le plus angoissant du siècle, la phtisie. Le développement des stations de curc dans l'arc alpin, qui commence dans le dernier tiers du siècle et se poursuit jusqu'à la seconde Giuerre mondiale, apparaît comme une application des théories géographico-médicales, associées au pragmatisme économique de certains médecins. La description et la comparaison typologique de quelques-unes des stations de cure édifiées dans l'arc alpin constituent un des aspects de notre projet. Dave I.üthi en donne ici une approche par l'histoire architecturale des sanatoriums. Ies formes architecturales, d'abord empruntées à des réalisations de type hôtelier et à des types pseudo-régionaux, se sont adaptées au terrain montagnard, aux pentes et aux conditions climatiques, et ont évolué vers une adéquation aux fonctions cliniques qu'on leur demandait de remplir. Cette évolution voit naitre des réponses remarquables aux questions de l'insolation et de l'aération, par l'invention de balcons destinés aux bains d'air, de dispositifs en terrasses pour l'héliothérapie, de façades en gradin... Mais si, d'un côté, l'histoire de l'architecture peut faire voir la prégnance des éléments naturels, des pratiques spatiales et des besoins concrets de la cure dans l'aménagement construit des stations, l'analyse du discours médical met l'accent sur une autre sorte de construction: celle qu'opèrent les contraintes "disciplinaires" et discursives. En référence aux idées de Michel Foucault, Adrien Ciuignard montre de son côté la fonction des organisations discursives dans la mise sur picd d'une " méthode sanatoriale" (l'expression est du Dr Burnand) qui prescrit leur place aux individus et impose aux corps ses propres formes ontologiques. L'examen des déclarations médicales et des productions romanesques du Dr Burnand conduit Guignard à une position radicalement sceptique à propos des traitements par l'air. S'appuyant d'autre part sur la grande variété des sites retenus pour y implanter des sanatoriums, il met en doute la pertinence de la fonction " altitude " et de la localisation alpine en général.

On ne doit pas pour autant identifier la cure d'air, les thérapies d'altitude et l'enthousiasme parfois douteux qu'elles ont suscité, avec la seule histoire de la tuberculose. Marc Piccand rappelle les recherches effectuées par les physiologistes à propos du mal des montagnes et analyse un aspect particulier de ces travaux, qui éclaire la valeur affective et sensible prêtée

4. Pour une vision d'ensemble de la recherche et de ses résultats, voir l'ouvrage le Bon Air des Alpes, sous la direction de Claude Reichler, à paraître. 
aux séjours dans les Alpes. Certains médecins, de même que des alpinistes, ont conçu l'altitude comme un "déterminant du paysage " dont l'influence bénéfique s'exercerait sur les malades au travers du sens cénesthésique - lequel implique l'ensemble des sensations et la vie psychique tout entière - bien plus qu'à travers les modifications apportées à l'organisme lui-même par la diminution de la pression, la sécheresse ou la raréfaction de l'oxygène. On sort ici du scientisme pour aller vers des conceptions impliquant l'individu comme sujet d'émotions, apte à établir des liens vivants et profonds avec son environnement, selon l'argumentation du Dr Hellpach dans son ouvrage de 1911, Géopsyché. Par la mise en évidence des discussions internes à la physiologie, notre projet fait voir un aspect des effets attribués à l'air des montagnes, qui s'écarte tout à fait du domaine des pathologies pulmonaires. I.e bien-être, les excitations sensorielles positives, les sensations d'appartenance au paysage, et jusqu'aux états mentaux bénéfiques ont été portés au crédit du bon air des Alpes non seulement par les poètes et les guides touristiques, mais aussi par les physiologistes. Leurs expérimentations et leurs théories ont conduit dans les Alpes tout un public désireux d'améliorer sa santé physique et mentale et de se reposer des fatigues de la vie urbaine. C'est précisément cette orientation physiologique des propriétés de l'air d'altitude qui, après avoir côtoyé les thérapies pulmonaires, a survécu à la découverte des antibiotiques et à la désaffection des sanatoriums. Après une période d'hésitation, et depuis les années 1980, un courant important du tourisme alpin est drainé par les thèmes du bien-être, du wellness comme dit la mode aujourd'hui. Dans sa contribution, Rafael Matos examine " ce qui reste " du thème du bon air dans le tourisme récent, et montre qu'en fait, le thème a la vie dure, si dure qu'il est là encore tout entier dans la promotion touristique comme dans les motivations des visiteurs des Alpes. Pourtant, les nouveaux usages des vertus curatives et des propriétés bénéfiques attribuées à l'air des montagnes sont aujourd'hui en conflit avec la mobilité touristique, qui engendre, elle, nuisances et pollution dans les espaces mêmes où l'on vient chercher la détente et la pure nature... Apparaît alors clairement la dimension contemporaine du problème de l'air, qui prend des aspects inquiétants, d'autant qu'il est lié à la concentration des gaz à effet de serre dans l'atmosphère et aux changements climatiques. Bien que notre projet se situe dans le cadre des sciences sociales, il ne se développe pas dans l'ignorance des recherches effectuées dans les sciences naturelles. Il faudrait au contraire qu'une coopération puisse être instituée entre les sciences historiques, les sciences naturelles et les acteurs du tourisme alpin de demain. Une perspective globale apparaît aujourd'hui indispensable à une juste évaluation, non pas seulement des bienfaits, mais aussi des menaces qui risquent de modifier autant la réalité que les représentations et les usages de l'air des montagnes. 